\title{
The relationship between seminal leukocytes, oxidative status in the ejaculate, and apoptotic markers in human spermatozoa
}

\author{
Cleyson Mupfiga, David Fisher, Thinus Kruger and Ralf Henkel
}

\begin{abstract}
The aim of this study was to investigate the relationship between seminal leukocytes, reactive oxygen species (ROS) production in the ejaculate, and markers of apoptosis in human spermatozoa. Semen samples were collected from 60 patients attending fertility clinics at the Reproductive Biology Unit at Tygerberg Academic Hospital and Vincent Pallotti Hospital, Cape Town, South Africa. The concentration of seminal leukocytes was determined and was correlated with ROS production in the ejaculate, the percentage of superoxide $\left(\cdot \mathrm{O}_{2}\right)$ and hydrogen peroxide $\left(\mathrm{H}_{2} \mathrm{O}_{2}\right)$-positive spermatozoa, glutathione activation in the ejaculate, and with markers of apoptosis in spermatozoa, namely cysteine-dependent aspartate-directed proteases (caspase)-3/7 activation, mitochondrial membrane potential $\left(\Delta \Psi_{\mathrm{m}}\right)$, and the percentage of terminal deoxynucleotidyl transferase-mediated dUTP-biotin nick end labeling (TUNEL)-positive sperm. Significant correlations with the concentration of seminal leukocytes were found for ROS production in the ejaculate, the percentage of $\mathrm{O}_{2}$-positive spermatozoa, and caspase-3/7 activation in the ejaculate. Leukocytospermic samples showed significantly higher ROS production, percentage of $\cdot \mathrm{O}_{2}$-positive sperm, GSH activation, and caspase-3/7 activation compared to non-leukocytospermic samples. The percentage of $\mathrm{O}_{2}$-positive sperm was significantly correlated with sperm $\Delta \Psi_{\mathrm{m}}$ and caspase-3/7 activation in the ejaculate. Sperm $\Delta \Psi_{\mathrm{m}}$ and TUNEL-positive sperm did not correlate with seminal leukocyte concentration. Data demonstrate that high seminal leukocyte concentrations that leads to increased seminal ROS production, and is also associated with caspase activation in the male germ cell and increased mitochondrial ROS production. The latter could possibly be a result of disturbed $\Delta \Psi_{\mathrm{m}}$. The activation of caspase$3 / 7$ could then follow the increased intrinsic superoxide levels due to depleted intrinsic glutathione (GSH). These cellular events might not directly and immediately lead to DNA fragmentation as an endpoint of apoptosis because of topological hindrances.
\end{abstract}

\section{Introduction}

Apoptosis is generally recognized as the underlying mechanism controlling the proliferation of germ cells during normal spermatogenesis [Print and Loveland 2000]. In mature human spermatozoa, however, the role of apoptosis is still controversial. Gorczya et al. [1993] were the first who reported the presence of DNA strand breaks in 
human ejaculated spermatozoa indicating the analogy to apoptosis in somatic cells. Since then, several studies have demonstrated the presence of morphological and biochemical markers of apoptosis in mature spermatozoa such as plasma membrane translocation of phosphatidylserine (PS) [Oosterhuis et al. 2000], disrupted mitochondrial membrane potential $\left(\Delta \Psi_{\mathrm{m}}\right)$ [Barroso et al. 2006], DNA fragmentation [Sakkas et al. 2002; Henkel et al. 2004], and cysteine-dependent aspartate-directed proteases (caspase) activation [Grunewald et al. 2005]. Despite this growing evidence of apoptosis in ejaculated spermatozoa, its implications for sperm fertilizing potential and assisted reproduction are still poorly understood. Male fertility appears to be negatively correlated with increased levels of sperm apoptotic markers [Wang et al. 2003]. Furthermore, the percentage of spermatozoa with DNA fragmentation, a late feature and hallmark of apoptosis, may negatively correlate with fertilization rates in in vitro fertilization and intracytoplasmic sperm injection [Henkel et al. 2003a; Henkel et al. 2004]. There is no consensus on the origins of apoptosis in ejaculated spermatozoa. On one hand, according to Sakkas et al. [1999], apoptosis is predetermined during testicular development following defective spermatogenesis. On the other hand, mitochondria are highly susceptible to external apoptotic agonists such as oxidative stress, and scientists are increasingly focusing on the role of increased production of reactive oxygen species (ROS) in cellular events related to apoptosis in sperm. A common cause of excessive ROS production in semen is male genital tract infection [Henkel et al. 2003b], where activated seminal leukocytes produce ROS and cytokines [Ochsendorf 1999]. While some authors have reported no significant difference in sperm apoptotic events between leukocyte-contaminated groups and their healthy counterparts [Ricci et al. 2002], others have demonstrated increased levels of apoptotic markers such as PS externalization after incubation with leukocytes and bacteria [Villegas et al. 2005], and caspase activation after incubation with Chlamydia trachomatis [Eley et al. 2005].

In male genital tract infection, activated leukocytes generate excessive ROS and secrete cytokines that have been negatively correlated with fertility [Depuydt et al. 1998] and semen quality [Eggert-Kruse et al. 2001]. The highly oxidative ROS cocktail released by leukocytes, containing hydroxyl radical $\left(\mathrm{OH}^{\bullet}\right)$, superoxide $\left(. \mathrm{O}_{2}^{-}\right)$, or the persistent hydrogen peroxide $\left(\mathrm{H}_{2} \mathrm{O}_{2}\right)$, can induce lipid peroxidation with the subsequent membrane and functional damages (for review: [Henkel 2011]) and substantial sperm DNA fragmentation [Alvarez et al. 2002], especially in infertile patients with deficient antioxidant capacity [Pasqualotto et al. 2000].

The prevalence of male infertility related to male genital tract infection varies from $10 \%$ to 35\% [World Health Organization 1999; Henkel et al. 2007]. If those men suffering from prostatitis at some point in their lives are included, the prevalence increases to $50 \%$ with the condition becoming chronic in 10\% [Schaeffer 2003]. Therefore, one can assume that in cases of longstanding male genital tract infection and inflammation such as orchitis, epididymitis [Weidner et al. 1999] and even prostatitis [Henkel et al. 2006], 
spermatozoa are directly exposed to activated leukocytes and their products for extended periods during their maturation. It is plausible then, that seminal leukocytes not only compromise sperm motility and decrease their fertilizing potential, but may also induce apoptosis in human spermatozoa.

The aim of this study was to investigate the relationship between the presence of leukocytes in the ejaculate and markers of apoptosis in spermatozoa, namely DNA fragmentation, $\Delta \Psi \mathrm{m}$, and caspase- $3 / 7$ activation in the ejaculate.

\section{Results}

Table 1 depicts the summary statistics of the parameters investigated in this study using a series of assays to determine mitochondrial membrane potential and superoxide production in sperm as illustrated in Figure 1. In Table 2, all correlations of the seminal leukocyte concentration with other parameters that were investigated in the study are summarized. As expected, significant, positive relationships of the seminal leukocyte concentration with the seminal ROS production $(\mathrm{r}=0.378 ; P=0.0064)$ and the sperm production of superoxide $(\mathrm{r}=0.336 ; P=0.0098)$ were observed. Furthermore, a highly significant strong positive correlation $(\mathrm{r}=0.527 ; P<0.0001)$ with caspase- $3 / 7$ in the ejaculate was found. However, all other associations of the seminal leukocyte concentration with the other parameters investigated in this study, including the percentage of DNA-damaged spermatozoa as determined by means of the TUNEL assay, as well as the percentage of sperm with disturbed $\Delta \Psi_{\mathrm{m}}$, showed no significant relationship.

Table 3 shows the correlations between different markers of seminal and sperm oxidative stress (seminal ROS production, $\mathrm{H}_{2} \mathrm{O}_{2}$-positive sperm, $\cdot \mathrm{O}_{2}$-positive sperm, and GSH activation in sperm), and sperm apoptotic markers (sperm with disturbed $\Delta \Psi_{\mathrm{m}}$, caspase-3/7 activation in the ejaculate, and TUNEL-positive sperm). Obviously, seminal ROS production was highly significantly and positively correlated with caspase$3 / 7$ activation in the ejaculate $(\mathrm{r}=0.487 ; P=0.0005)$, but to a lesser extent with sperm DNA damage $(\mathrm{r}=0.331 ; P=0.0171)$. However, no relationship could be found with the percentage of spermatozoa showing disturbed $\Delta \Psi \mathrm{m}$. Interestingly there was also no correlation between the percentage of $\mathrm{H}_{2} \mathrm{O}_{2}$-positive sperm and any of the apoptotic parameters. In comparison, the percentage of ${ }^{-} \mathrm{O}_{2}$ positive sperm correlated positively with disturbed sperm $\Delta \Psi_{\mathrm{m}} \quad(\mathrm{r}=0.261 ; P=0.0446)$. 


\begin{tabular}{lcccc}
\hline & $\mathrm{n}$ & Mean \pm SD & Median & Range \\
\hline Sperm count $\left(\times 10^{6} / \mathrm{mL}\right)$ & 60 & $76.8 \pm 57.6$ & 80 & $8-338$ \\
Sperm motility $(\%)$ & 59 & $52.5 \pm 10.6$ & 50 & $20-70$ \\
Peroxidase-positive cells $\left(\times 10^{6} / \mathrm{mL}\right)$ & 60 & $0.52 \pm 1.28$ & 0.14 & 0.43 \\
ROS production in the ejaculate $\dagger$ & 53 & $10.6 \pm 41.7$ & 20.4 & $-0.5-248$ \\
$\mathrm{H}_{2} \mathrm{O}_{2}$-positive sperm (DCF) $(\%)$ & 60 & $23.5 \pm 13.7$ & 21.0 & $5.4-89.4$ \\
$\mathrm{O}_{2}^{-}$-positive sperm (DHE) $(\%)$ & 60 & $25.9 \pm 15.8$ & 18.2 & $4.1-76.9$ \\
$\mathrm{GSH}$ activation in sperm $\dagger$ & 53 & $73.2 \pm 182$ & 16.8 & $-21-1187$ \\
$\Delta \psi_{\mathrm{m}}$-negative sperm $(\%)$ & 60 & $25.2-20.1$ & 133.8 & $4.5-83.9$ \\
Caspase-3/7 activation in sperm $\dagger$ & 58 & $247 \pm 353$ & 9.2 & $44-1842$ \\
TUNEL-positive sperm $(\%)$ & 59 & $10.3 \pm 6.6$ & $2.3-37.1$ \\
\hline
\end{tabular}

ROS: reactive oxygen species; $\mathrm{H}_{2} \mathrm{O}_{2}$ : hydrogen peroxide; DCF: dichlorofluorescein; $\cdot \mathrm{O}_{2}^{-}$: superoxide anion; DHE: dihydroethidium; GSH: glutathione; $\Delta \psi_{\mathrm{m}}$ : mitochondrial membrane potential; Caspase: cysteine-dependent aspartate-directed proteases; TUNEL: terminal deoxynucleotidyl transferase-mediated dUTP-biotin nick end labeling. $\dagger \times 10^{4}$ RLU/20 $\times 10^{6}$ sperm.
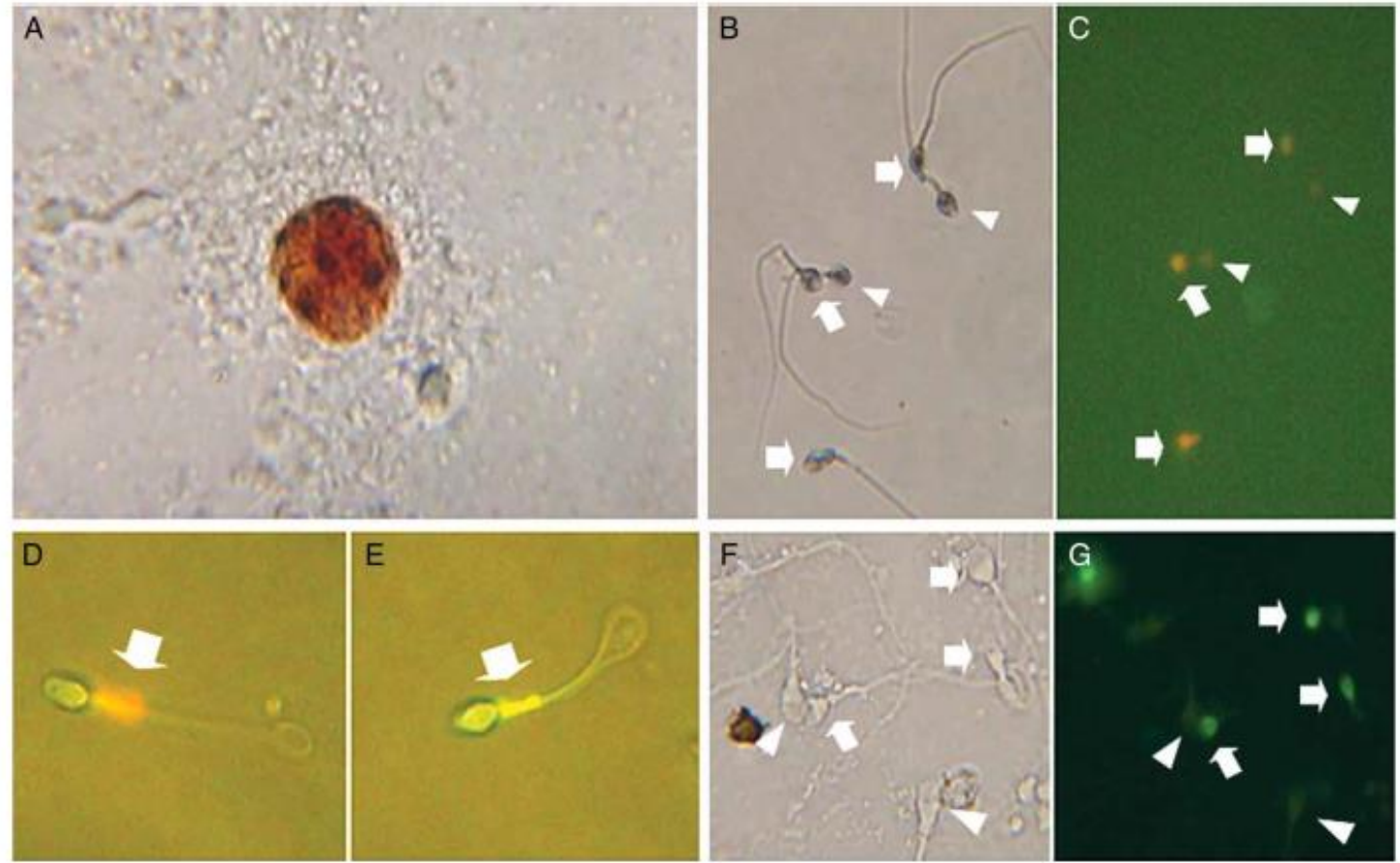

Figure 1. Positive peroxidase stain to detect peroxidase-positive cells. A) Dihydroethidium (DHE) to test for superoxide anion (. $\mathrm{O}_{2}^{-}$) in sperm (B and $\mathrm{C}$ ) $\cdot \mathrm{O}_{2}^{-}$-positive sperm fluoresced orange (arrows), $\cdot \mathrm{O}_{2}$-negative sperm show no or only faint fluorescence (arrow head). DePsipher; sperm with intact (D) and disturbed mitochondrial membrane potential $(\Delta \psi \mathrm{m})(\mathrm{E})$. Terminal deoxynucleotidyl transferase-mediated dUTP-biotin nick end labeling (TUNEL) to measure sperm DNA fragmentation; sperm with damaged DNA emitted a bright green fluorescence (F and G, arrows), TUNEL-negative sperm show no or only faint fluorescence (F and G, arrow head).

This correlation was even much more pronounced with caspase- $3 / 7$ activation $(\mathrm{r}=$ $0.457 ; P=0.0005)$. Whereas the latter two parameters also showed significant positive correlations with the activation of sperm GSH, no association was found with sperm DNA damage. Furthermore, GSH activation was significantly positively $(\mathrm{r}=0.577 ; P<$ o.0o01) associated with extrinsic seminal ROS production.

Semen samples were grouped according to the concentration of seminal leukocytes into three subgroups; < $0.1 \times 10^{6} / \mathrm{mL}$ (low leukocyte; $\mathrm{n}=21$ ), $0.1-0.9 \times 10^{6} / \mathrm{mL}$ (medium leukocyte; $\mathrm{n}=31$ ), and $1 \times 10^{6} / \mathrm{mL}$ or greater $(\mathrm{n}=8)$. The eight samples with $1 \times 10^{6}$ or more leukocytes $/ \mathrm{mL}$ of semen were considered to be leukocytospermic 
[World Health Organization 1999]. Table 4 presents the comparison of markers of oxidative stress and apoptosis amongst the three subgroups. Reactive oxygen species production, the percentage of $\cdot \mathrm{O}^{-}$-positive sperm, GSH activation, and caspase- $3 / 7$ activation were significantly higher in the leukocytospermic group compared to the other two groups. Except for the GSH activation, the medium leukocyte group (o.1$0.9 \times 10^{6} / \mathrm{mL}$ ) showed higher, but not significantly higher, values for parameters directly associated with oxidative stress, namely ROS in the ejaculate, the percentages of $\cdot \mathrm{O}^{-}$-positive sperm, and caspase-3/7 activation, respectively, than the low leukocyte group $\left(<0.1 \times 10^{6} / \mathrm{mL}\right)$. In addition, the Kruskal-Wallis test shows significant, concentration-dependent relationships between seminal leukocytes and seminal ROS production, the percentages of $\cdot \mathrm{O}^{-}$-positive sperm, GSH activation, and caspase-3/7 activation, respectively (Table 4). For the other parameters measured, no difference could be observed.

Table 2. Correlations of the concentration of seminal leukocytes with markers of seminal oxidative stress, and sperm apoptotic parameters.

\begin{tabular}{lllr}
\hline & $\mathrm{N}$ & \multicolumn{1}{c}{$\rho$} & $P$-value \\
\hline ROS production in the ejaculate $\dagger$ & 53 & 0.378 & 0.0064 \\
$\mathrm{H}_{2} \mathrm{O}_{2}$-positive sperm (DCF) (\%) & 60 & 0.085 & 0.5162 \\
$\cdot \mathrm{O}_{2}^{-}$-positive sperm (DHE) (\%) & 60 & 0.336 & 0.0098 \\
$\mathrm{GSH}$ activation in sperm $\dagger$ & 53 & 0.211 & 0.1275 \\
Sperm with disturbed $\Delta \psi_{\mathrm{m}}(\%)$ & 60 & 0.0854 & 0.5121 \\
Caspase-3/7 activation in sperm $\dagger$ & 58 & 0.527 & $<0.0001$ \\
TUNEL-positive sperm $(\%)$ & 59 & 0.0195 & 0.8817 \\
\hline
\end{tabular}

ROS: reactive oxygen species; $\mathrm{H}_{2} \mathrm{O}_{2}$ : hydrogen peroxide; DCF: dichlorofluorescein; $\cdot \mathrm{O}_{2}^{-}$: superoxide anion; DHE: dihydroethidium; GSH: glutathione; $\Delta \psi_{\mathrm{m}}$ : mitochondrial membrane potential; Caspase: cysteine-dependent aspartate-directed proteases; TUNEL: terminal deoxynucleotidyl transferase-mediated dUTP-biotin nick end labeling. $\dagger \times 10^{4} \mathrm{RLU} / 20 \times 10^{6}$ sperm.

\section{Discussion}

The role played by leukocytes in the human ejaculate is not fully understood and thus subject to much controversy. However, there is consensus that high concentrations of leukocytes, exceeding $1 \times 10^{6}$ cells/mL, are harmful to spermatozoa and impair male fertility. 
Table 3. Correlations of markers of seminal oxidative stress with sperm apoptotic markers.

\begin{tabular}{|c|c|c|c|}
\hline \multirow[b]{2}{*}{ Markers of oxidative stress } & \multicolumn{3}{|c|}{ Markers of apoptosis } \\
\hline & Sperm with disturbed $\Delta \psi_{\mathrm{m}}(\%)$ & Caspase- $3 / 7$ activation in sperm $\dagger$ & TUNEL-positive sperm (\%) \\
\hline \multirow[t]{2}{*}{ ROS production in the ejaculate $\dagger$} & $r=0.175$ & $\mathrm{r}=0.487$ & $\mathrm{r}=0.331$ \\
\hline & $\mathrm{n}=53$ & $\mathrm{n}=52$ & $\mathrm{n}=53$ \\
\hline \multirow[t]{2}{*}{$\mathrm{H}_{2} \mathrm{O}_{2}$-positive sperm (DCF) (\%) } & $r=0.203$ & $\mathrm{r}=0.218$ & $r=0.146$ \\
\hline & $\mathrm{P}=0.1198$ & $\mathrm{P}=0.1004$ & $\mathrm{P}=0.2649$ \\
\hline \multirow[t]{3}{*}{$\cdot \mathrm{O}_{2}^{-}$-positive sperm (DHE) (\%) } & $r=0.261$ & $r=0.457$ & $\mathrm{r}=0.204$ \\
\hline & $\mathrm{P}=0.0446$ & $\mathrm{P}<0.0001$ & $\mathrm{P}=0.1207$ \\
\hline & $\mathrm{n}=60$ & $\mathrm{n}=58$ & $\mathrm{n}=59$ \\
\hline \multirow[t]{3}{*}{ GSH activation in sperm $\dagger$} & $r=0.385$ & $r=0.529$ & $r=0.205$ \\
\hline & $\mathrm{P}=0.0055$ & $\mathrm{P}=0.0002$ & $\mathrm{P}=0.1399$ \\
\hline & $\mathrm{n}=53$ & $\mathrm{n}=53$ & $\mathrm{n}=53$ \\
\hline
\end{tabular}

$\Delta \psi_{\mathrm{m}}:$ mitochondrial membrane potential; Caspase: cysteine-dependent aspartate-directed proteases; TUNEL: terminal deoxynucleotidyl transferase-mediated dUTP-biotin nick end labelling; ROS: reactive oxygen species; $\mathrm{H}_{2} \mathrm{O}_{2}$ : hydrogen peroxide; DCF: dichlorofluorescein; . $\mathrm{O}_{2}^{-}$: superoxide anion; DHE: dihydroethidium; GSH: glutathione. $\dagger \times 10^{4} \mathrm{RLU} / 20 \times 10^{6}$ sperm.

Table 4. Comparison of the subgroups of seminal leukocyte concentration.

\begin{tabular}{|c|c|c|c|c|}
\hline \multirow[b]{2}{*}{$\begin{array}{l}\text { Markers of oxidative } \\
\text { stress and apoptosis }\end{array}$} & \multicolumn{3}{|c|}{ Seminal leukocyte concentration subgroups } & \multirow[b]{2}{*}{$\begin{array}{c}\text { Kruskal- } \\
\text { Wallis (P-value) }\end{array}$} \\
\hline & $\begin{array}{c}\text { Leukocytes }<0.1 \times 10^{6} / \\
\text { mL (low leukocyte) }\end{array}$ & $\begin{array}{c}\text { Leukocytes } 0.1-0.9 \times 10^{6} / \\
\text { mL (medium leukocyte) }\end{array}$ & $\begin{array}{l}\text { Leukocytes > } 1 \times 10^{6} / \\
\text { mL (leukocytospermic) }\end{array}$ & \\
\hline ROS production & $0.59 \pm 1.19^{\mathrm{a}}$ & $3.29 \pm 10.17^{\mathrm{b}}$ & $77.17 \pm 107.37^{\mathrm{a}, \mathrm{b}}$ & 0.0005 \\
\hline in the ejaculate $\dagger$ & $\mathrm{n}=21$ & $\mathrm{n}=26$ & $\mathrm{n}=6$ & \\
\hline $\mathrm{H}_{2} \mathrm{O}_{2}$-positive & $22.4 \pm 10.9$ & $22.1 \pm 10.3$ & $32.1 \pm 26.3$ & 0.8553 \\
\hline sperm (DCF) (\%) & $\mathrm{n}=21$ & $\mathrm{n}=31$ & $\mathrm{n}=8$ & \\
\hline sperm (DHE) (\%) & $\mathrm{n}=21$ & $\mathrm{n}=31$ & $\mathrm{n}=8$ & \\
\hline GSH activation & $47.31 \pm 106.15^{\mathrm{e}}$ & $32.75 \pm 48.41^{\mathrm{f}}$ & $339.08 \pm 436.69^{\mathrm{e}, \mathrm{f}}$ & 0.0297 \\
\hline in sperm $\dagger$ & $\mathrm{n}=21$ & $\mathrm{n}=26$ & $\mathrm{n}=6$ & \\
\hline Sperm with & $23.2 \pm 19.0$ & $23.0 \pm 16.9$ & $39.0 \pm 30.1$ & 0.2551 \\
\hline disturbed $\Delta \psi_{\mathrm{m}}(\%)$ & $\mathrm{n}=21$ & $\mathrm{n}=31$ & $\mathrm{n}=8$ & \\
\hline Caspase $-3 / 7$ activation & $119.63 \pm 62.29^{\mathrm{g}}$ & $168.21 \pm 124.56^{\mathrm{h}}$ & $859.21 \pm 660.83^{\mathrm{g}, \mathrm{h}}$ & 0.0005 \\
\hline in sperm $\dagger$ & $\mathrm{n}=20$ & $\mathrm{n}=30$ & $\mathrm{n}=8$ & \\
\hline sperm $(\%)$ & $\mathrm{n}=21$ & $\mathrm{n}=30$ & $\mathrm{n}=8$ & \\
\hline
\end{tabular}

ROS: reactive oxygen species; $\mathrm{H}_{2} \mathrm{O}_{2}$ : hydrogen peroxide; DCF: dichlorofluorescein; $\cdot \mathrm{O}_{2}^{-}$: superoxide anion; DHE: dihydroethidium; GSH: glutathione; $\Delta \psi_{\mathrm{m}}$ : mitochondrial membrane potential; Caspase: cysteine-dependent aspartate-directed proteases; TUNEL: terminal deoxynucleotidyl transferase-mediated dUTP-biotin nick end labeling. $\dagger \times 10^{4} \mathrm{RLU} / 20 \times 10^{6}$ sperm. Values are mean \pm SD.

a-h Same letters indicate statistically significant differences $(P<0.05)$.

\section{The negative impact}

of seminal leukocytes is supported by data indicating that these phagocytic cells are the primary source of ROS in the ejaculate [Tremellen 2008], and leukocytospermic patients show abnormal semen profiles and defective sperm function [Aziz et al. 2004]. In this study, leukocytospermia was detected in $13.3 \%$ of the patients. This finding is in the range of those reported in the literature [Yanushpolsky et al. 1996; Zorn et al. 2010]. As expected, ROS production correlated significantly with leukocyte concentration, and leukocytospermic men had significantly higher levels of ROS production compared to their non-leukocytospermic counterparts. Seminal leukocyte concentration correlated positively with sperm superoxide production, a finding that was also reported by Zorn et al. [2010]. In addition, seminal leukocyte concentration was associated with caspase-3/7 activation in the ejaculate. This is in contrast to observations by Ricci et al. [2002] who found no correlation between seminal 
leukocytes and apoptosis. However, Ricci et al. [2002] used Annexin-V to detect apoptotic sperm and this probe can also be positive in acrosome-reacting sperm [Martin et al. 2005], whereas caspase- $3 / 7$ is a more specific probe.

The increased ROS production could possibly be a result of disturbed $\Delta \Psi_{\mathrm{m}}$. The activation of caspase- $3 / 7$ could then follow the increased intrinsic superoxide levels due to depleted intrinsic antioxidative glutathione. These cellular changes might not directly and immediately lead to DNA fragmentation as an endpoint of apoptosis because of topological hindrances, thus confirming the hypothesis by Aitken and Koppers [2011]. In turn, it can be speculated that sperm nuclear DNA damage as seen in this study might, then, rather be directly caused by large amounts of the membrane-permeable hydrogen peroxide produced by leukocytes (at least in patients with leukocyte counts less that $1 \times 10^{6} / \mathrm{ml}$ ), than that generated in male germ cells. This assumption is corroborated by the observations that the percentage of $\mathrm{H}_{2} \mathrm{O}_{2}$-positive sperm did not correlate with sperm DNA damage and that there is no difference between the three subgroups of patients with respect to $\mathrm{H}_{2} \mathrm{O}_{2}$-positivity. This might also be the reason why no relationship between sperm intrinsic superoxide production and DNA damage was observed. Curiously, there was no correlation between seminal leukocyte concentration and either the percentage of TUNEL-positive sperm indicating DNA fragmentation, or $\Delta \Psi_{\mathrm{m}}$-positive sperm.

The ability of spermatozoa to produce ROS has been recognized since the 1940 s [MacLeod 1943]. Reactive oxygen species originate from either sperm mitochondria [Koppers et al. 2008] or a membrane-bound nicotinamide adenine dinucleotide phosphate oxidase [Aitken et al. 1997]. This internally generated ROS has been linked to impaired motility [Koppers et al. 2008] and DNA fragmentation [Henkel et al. 2005]. Located in the midpiece of the spermatozoa, mitochondria produce the ATP necessary for the physiological functions. However, about $1-5 \%$ of the consumed oxygen is converted into free radicals [Boveris and Chance 1973] which are leaking out contributing to superoxide production in spermatozoa [Koppers et al. 2008]. The ROS produced via this mechanism are normally regarded as cytotoxic by-products that are involved in the etiology of disease and aging [Raha and Robinson 2000]. Besides the association with seminal leukocyte concentration, this study shows that sperm superoxide production is correlated with loss of sperm $\Delta \Psi_{\mathrm{m}}$, and caspase-3/7 activation indicating a role for seminal leukocyte-induced intrinsic ROS in sperm apoptotic events, involving the mitochondrial pathway.

Sperm mitochondria are susceptible to oxidative stress, cryopreservation, and increased intracellular calcium [Oehninger et al. 2003]. Mitochondria have also been associated with several key events of apoptosis including the release of cytochrome $\mathrm{c}$ and the loss of $\Delta \Psi_{\mathrm{m}}$ [Paasch et al. 2004]. The latter has been regarded as an early marker of apoptosis and is associated with abnormal semen parameters [Kasai et al. 2002; Marchetti et al. 2002] and poor fertility [Gallon et al. 2006]. Here, reduced $\Delta \Psi_{\mathrm{m}}$

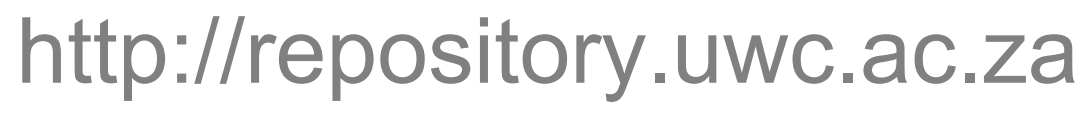


was correlated with sperm $\cdot \mathrm{O}_{2}^{-}$production and GSH activation. Given that this result shows positive correlation between sperm $\cdot \mathrm{O}_{2}^{-}$and seminal leukocyte concentration, this might indicate a (indirect) relationship between oxidative stress in the ejaculate, intrinsic ROS, and disrupted $\Delta \Psi_{\mathrm{m}}$ in sperm. Yet, no association between disrupted sperm $\Delta \Psi_{\mathrm{m}}$ and caspase-3/7 or DNA fragmentation could be observed.

Caspases are a group of aspartic acid-directed cysteine proteases that, upon cleavage, become activated and transduce apoptotic signals [Nicholson 1999]. Apoptosis-related caspases of all major subtypes have been found in spermatozoa [Paasch et al. 2004]. In this study, caspase-3/7 activation was correlated with seminal leukocyte concentration and ROS production as well as with sperm $\cdot \mathrm{O}_{2}^{-}$production.

DNA fragmentation has been well characterized in human spermatozoa [Evenson and Wixon 2006], and is associated with infertility, poor embryo development, and low pregnancy success rates during assisted reproduction [Sun et al. 1997; Lopes et al. 1998; Henkel et al. 2004]. Here, a positive correlation of sperm DNA fragmentation with seminal ROS production could be confirmed. Nevertheless, no correlation between intrinsic ROS production $\left(\mathrm{H}_{2} \mathrm{O}_{2}\right.$ or superoxide) and other markers of apoptosis was observed. In the general view of the apoptotic process, it might be anticipated that the culmination of the apoptotic cascade in spermatozoa would be the activation of endonucleases leading to DNA fragmentation and cell death [Aitken and Koppers 2011]. However, in spermatozoa this process is restricted by the location of the mitochondria and the nucleus in different cellular compartments as well as the highly condensed nature of the chromatin in ejaculated spermatozoa [Aitken and Koppers 2011]. As a result of these physical constraints, apoptosis in spermatozoa may not be immediately followed by DNA fragmentation. Furthermore, some DNA fragmentation in spermatozoa may be a result of unresolved DNA strand breaks during spermiogenesis [Sakkas et al. 2002], and abnormal chromatin condensation [De Iuliis et al. 2009]. Thus, results of this study suggest that sperm DNA fragmentation may rather be a consequence of exposure to excessive ROS in the ejaculate emanating from infiltrating leukocytes than of apoptosis.

Oxidative stress of spermatozoa can either result from excessive exposure of male germ cells to ROS or be a consequence of deficient antioxidative defences. Since male germ cells have very little intrinsic antioxidant capacity because they have shed most of their cytoplasm, they possess extremely low levels of cytoplasm antioxidant enzymes such as glutathione peroxidase, catalase, and superoxide dismutase. Furthermore, the little cytoplasm is largely restricted to the midpiece; this offers scanty protection to the large area of plasma membrane overlying the sperm head and tail. In this study, GSH activation was correlated to ROS production in the ejaculate, loss of sperm $\Delta \Psi_{\mathrm{m}}$, and caspase-3/7 activation. This indicates that increased reduction of glutathione in the ejaculate is linked to mitochondrial damage and caspase activation. 
In conclusion, this study demonstrates that the increased concentration of seminal leukocyte are not only the cause for increased seminal ROS production, but proposes that seminal leukocytes can trigger caspase activation in the male germ cell as well as an increased intrinsic ROS production in spermatozoa. The discrepancy between the occurrence of early signs of apoptosis and the lack of its execution in terms of DNA fragmentation might then be due to topological hindrances.

\section{Materials and Methods}

A total of 60 semen samples were obtained from patients attending the Reproductive Biology Unit at Tygerberg Academic Hospital, and Vincent Palotti Hospital in Cape Town, South Africa. This study was approved by the Institutional Review Boards of the Stellenbosch University and the University of the Western Cape, and all participants granted informed consent. The semen samples were produced by masturbation into sterile containers after $2-5 \mathrm{~d}$ of sexual abstinence. After complete liquefaction in an incubator at $37^{\circ} \mathrm{C}$ within 30 to $60 \mathrm{~min}$, sperm concentration and motility were evaluated by experienced lab technicians according to WHO criteria [WHO 1999].

\section{Determination of leukocytes}

The Endtz test was used to evaluate the concentration of seminal leukocytes [Shekarriz et al. 1995]. In brief, $20 \mu \mathrm{L}$ of liquefied semen were placed in a $1.5 \mathrm{~mL}$ Eppendorf tube (Greiner Bio-One $\mathrm{GmbH}$, Frickenhausen, Germany), followed by 20 $\mu \mathrm{L}$ of PBS ( $\mathrm{pH} 7.0)$ and $40 \mu \mathrm{L}$ of benzidine stock solution $(0.0125 \% \mathrm{w} / \mathrm{v}$ benzidine, Sigma, St Louis, MO, USA, in 50\% ethanol). The mixture was mixed and allowed to incubate for $5 \mathrm{~min}$ at room temperature. Peroxidase-positive leukocytes stained dark brown and were counted in a Makler chamber (Sefi Medical Instruments, Haifa, Israel) under a bright-field at $\times 400$ magnification (Fig. 1A). For calculations, semen samples were grouped according to their seminal leukocyte concentrations into 3 subgroups: < $0.1 \times 10^{6} / \mathrm{mL}$ (low leukocyte; $\mathrm{n}=21$ ), $0.1-0.9 \times 10^{6} / \mathrm{mL}$ (medium leukocyte; $\mathrm{n}=31$ ), and $>1 \times 10^{6} / \mathrm{mL}$ (leukocytospermic; $\mathrm{n}=8$ ) [World Health Organization 1999].

\section{Determination of ROS production}

For the determination of extrinsic ROS production, aliquots of liquefied semen were centrifuged at $300 \times g$ for $10 \mathrm{~min}$. After seminal plasma was discarded, the pellet was washed twice in a human tubal fluid medium containing $1 \%$ human serum albumin (HTF-HSA; [Quinn et al. 1985]) and adjusted to a concentration of $20 \times 10^{6}$ sperm $/ \mathrm{mL}$. Levels of ROS were determined by a chemiluminescence assay using luminol (5-amino-2,3 dihydro-1,4 phalazinedione; Sigma) as described by Shekarriz et al. [1995]. Chemi-luminescence was measured using a Berthold luminometer (MicroLumat Plus LB96V Bad-Wildbad, Germany). The luminometer was set in the integration mode at $37^{\circ} \mathrm{C}$ and injected $25 \mu \mathrm{L}$ of luminol to $250 \mu \mathrm{L}$ of sperm suspension, blank (HTF-HSA only) and control (HTF-HSA and luminol). After reading for $10 \mathrm{~min}$, 
background reading was subtracted and the result expressed as relative light units (RLU) per $20 \times 10^{6}$ sperm $/ \mathrm{mL}$.

\section{Determination of sperm intracellular $\mathrm{H}_{2} \mathrm{O}_{2}$}

Since chemiluminescence methods have poor specificity to $\mathrm{H}_{2} \mathrm{O}_{2}$ [Myhre et al. 2003], 2,7-dihydrofluorescein acetate ( $\mathrm{H}_{2}$ DCFDA; Molecular Probes, Eugene, OR, USA), a stable non-fluorescent cell-permeable probe that de-esterifies in the presence of intracellular $\mathrm{H}_{2} \mathrm{O}_{2}$ to form a fluorescent 2,7-dichlorofluorescein (DCF) was used. DCF emits a fluorescence at $530 \mathrm{~nm}$ in response to $488 \mathrm{~nm}$ excitation. Aliquots of liquefied semen were washed in PBS $(\mathrm{pH} 7.4)$ and centrifuged at $300 \times g$ for $10 \mathrm{~min}$. After discarding the supernatant, the pellet was re-suspended in a solution containing $2.5 \mu \mathrm{M}$ and incubated for $45 \mathrm{~min}$ at $37^{\circ} \mathrm{C}$ in the dark. The sample was analyzed using a fluorescent microscope (Zeiss; Oberkochen, Germany; $\times 400$ magnification) by counting at least 200 spermatozoa. Sperm fluorescing bright green were regarded as $\mathrm{H}_{2} \mathrm{O}_{2}$-positive, and the results were expressed as a percentage of total sperm counted.

\section{Determination of mitochondrial superoxide production in sperm}

Sperm production of superoxide $\left(\cdot \mathrm{O}^{-}\right)$by the mitochondria was evaluated using dihydroethidium (DHE; Molecular Probes) according to Henkel et al. [2005]. For the assay, aliquots of liquefied semen were washed in PBS and centrifuged at $300 \times g$ for 10 min. The supernatant was discarded, and the pellet was re-suspended with DHE solution $(2 \mu \mathrm{M})$ and then incubated in the dark at $37^{\circ} \mathrm{C}$ for $15 \mathrm{~min}$. Cells were then washed once $(300 \times g$ for $5 \mathrm{~min})$ and at least 200 spermatozoa were analyzed with a fluorescence microscope (Zeiss; $\times 400$ magnification) using a $590 \mathrm{~nm}$ band pass filter. Sperm that fluoresced red (.O -positive) were recorded as a percentage of all the sperm observed (Fig. 1B,C).

Determination of glutathione activation in the ejaculate The GSH-Gloтм luminescence assay kit (Promega, Madison, WI, USA) was used to estimate the activation of reduced glutathione in the ejaculate according to the manufacturer's specifications. In brief, 50 $\mu \mathrm{L}$ of GSH-Gloтм reagent was added to $50 \mu \mathrm{L}$ of washed semen (diluted with PBS, centrifuged at $300 \times \mathrm{g}$ for $10 \mathrm{~min}$ ) adjusted to $20 \times 10^{6} \mathrm{sperm} / \mathrm{mL}$, and incubated at room temperature for $30 \mathrm{~min}$ in the dark. Subsequently, $50 \mu \mathrm{L}$ of luciferin detection reagent was added and the mixture was incubated at room temperature for $15 \mathrm{~min}$ in the dark. Chemiluminescence was measured using a Berthold luminometer set in the integration mode at $37^{\circ} \mathrm{C}$. Readings were taken from wells containing $100 \mu \mathrm{L}$ of the reaction mixture and sperm suspension, blank (PBS only), and control (PBS and reaction mixture). After reading for $10 \mathrm{~min}$, background reading was subtracted and the result expressed as RLU per $20 \times 10^{6}$ sperm $/ \mathrm{mL}$.

Determination of sperm mitochondrial membrane potential Sperm mitochondrial membrane potential $\left(\Delta \Psi_{\mathrm{m}}\right)$ was determined using a lipophilic cation DePsipherтм

\section{http://repository.uwc.ac.za}


(5,5',6,6'-tetra-chloro-1,1',3,3'-tetraethylbenzimidazoyl carbocyanin iodide; R\&D Systems, Trevigen, Minneapolis, USA). The probe was prepared according to the manufacturer's recommendation, and was added to aliquots of washed semen and incubated at $37^{\circ} \mathrm{C}$ for $20 \mathrm{~min}$ in the dark. At least 200 spermatozoa were analyzed under a fluorescence microscope (Zeiss; $530 \mathrm{~nm}$ band pass filter $\times 400$ magnification). The number of green-fluorescing sperm indicating disturbed $\Delta \Psi_{\mathrm{m}}$ was recorded as a percentage of the total sperm evaluated (Fig. 1D,E).

Determination of caspase-3/7 activation in the ejaculate Caspase activation was evaluated using Caspase-Gloтм3/7 assay kit (Promega). The Caspase-Gloтм reagent was prepared to the recommendations of the manufacturer. For the assay, $100 \mu \mathrm{L}$ of the reagent was added to $100 \mu \mathrm{L}$ of semen that had been washed in PBS ( $\mathrm{pH} 7.4$ ), centrifuged for 10 $\min$ at $300 \times \mathrm{g}$, and adjusted to $20 \times 10^{6}$ sperm $/ \mathrm{mL}$. The mixture was incubated for one $\mathrm{h}$ at room temperature in the dark and reading were taken using a Berthold luminometer set at integration mode. Readings were taken from wells containing $100 \mu \mathrm{L}$ of the reaction mixture and sperm suspension, blank (PBS only), and control (PBS and reaction mixture). After reading for $10 \mathrm{~min}$, background reading was subtracted and the result expressed as RLU per $20 \times 10^{6}$ sperm $/ \mathrm{mL}$.

\section{Determination of sperm DNA fragmentation}

For the determination of sperm DNA fragmentation, the terminal deoxynucleotidyl transferase-mediated dUTP-biotin nick end labeling (TUNEL) assay as described previously [Henkel et al. 2003a] was employed. Semen aliquots were centrifuged at 300 $\times \mathrm{g}$ for $10 \mathrm{~min}$, the supernatant was discarded and the pellet re-suspended in PBS. A droplet of this sperm suspension was smeared onto a StarFrostrm slide (Knittel Gläser, Braunschweig, Germany), air-dried, and fixed by immersing in freshly prepared $4 \%$ methanol-free formaldehyde in PBS at $4^{\circ} \mathrm{C}$ for $25 \mathrm{~min}$. Afterwards, the slides were washed in fresh PBS for $5 \mathrm{~min}$ at room temperature, treated with $0.2 \%$ Triton $\mathrm{Tm}$ X-100 (Sigma) in PBS for $5 \mathrm{~min}$, and then rinsed twice in PBS for $5 \mathrm{~min}$ at room temperature. The reagents of the TUNEL reagent (DeadEndтм, Promega) were prepared and the final steps of the procedure were performed according to the manufacturer's instructions. The samples were analyzed with a fluorescent microscope (Zeiss, $530 \mathrm{~nm}$ band pass filter) at $\times 400$ magnification. The percentage of sperm showing bright green fluorescence (TUNEL-positive) was determined (Fig. 1F,G).

\section{Statistical analysis}

All statistical calculations were performed with appropriate tests after checking the normality of the data by means of the MedCalc ${ }^{\circledR}$ statistical software (Version 11.5.1, Maria-kerke, Belgium). The Mann-Whitney and Kruskal-Wallis tests were used to directly compare values and variables with two or more levels, respectively. Correlations were determined by the Spearman test. A $P$-value of $P<0.05$ was regarded as significant for all tests.

\section{http://repository.uwc.ac.za}


Declaration of interest:Theauthorsdeclarethattheydonot have any conflict of interest.

Author contributions: Designing of the study: RH, CM, TK; Executing of the study: CM, RH; Evaluation of data: CM, RH, DF; Conceptualization: CM, RH, DF; Writing of the paper: CM, RH. 


\section{References}

Aitken, R.J., Fisher, H.M., Fulton, N., Gomez, E., Knox, W., Lewis, B., et al. (1997) Reactive oxygen species generation by human spermatozoa is induced by exogenous NADPH and inhibited by the flavoprotein inhibitors diphenylene iodonium and quinacrine. Mol Reprod Dev 47:468-482.

Aitken, R.J. and Koppers, A.J. (2011) Apoptosis and DNA damage in human spermatozoa. Asian J Androl 13:36-42.

Alvarez, J.G., Sharma, R.K., Ollero, M., Saleh, R.A., Lopez, M.C., Thomas, A.J., Jr. et al. (2002) Increased DNA damage in sperm from leukocytospermic semen samples as determined by the sperm chromatin structure assay. Fertil Steril 78:319-329.

Aziz, N., Saleh, R.A., Sharma, R.K., Lewis-Jones, I., Esfandiari, N., Thomas, A.J., et al. (2004) Novel association between sperm reactive oxygen species production, sperm morphological defects, and the sperm deformity index. Fertil Steril 81:349-354.

Barroso, G., Taylor, S., Morshedi, M., Manzur, F., Gavino, F. and Oehninger, S. (2006) Mitochondrial membrane potential integrity and plasma membrane translocation of phosphatidylserine as early apoptotic markers: a comparison of two different sperm subpopulations. Fertil Steril 85:149-154.

Boveris, A. and Chance, B. (1973) The mitochondrial generation of hydrogen peroxide. General properties and effect of hyperbaric oxygen. Biochem J 134:707-716.

De Iuliis, G.N., Thomson, L.K., Mitchell, L.A., Finnie, J.M., Koppers, A.J., Hedges, A., et al. (2009) DNA damage in human spermatozoa is highly correlated with the efficiency of chromatin remodeling and the formation of 8-hydroxy-20-deoxyguanosine, a marker of oxidative stress. Biol Reprod 81:517-524.

Depuydt, C., Zalata, A., Christophe, A., Mahmoud, A. and Comhaire, F. (1998) Mechanisms of sperm deficiency in male accessory gland infection. Andrologia 30:29-33.

Eggert-Kruse, W., Boit, R., Rohr, G., Aufenanger, J., Hund, M. and Strowitzki, T. (2001) Relationship of seminal plasma interleukin (IL)-8 and IL-6 with semen quality. Hum Reprod 16:517-528.

Eley, A., Hosseinzadeh, S. and Hakimi, H. (2005) Apoptosis of ejaculated human sperm is induced by co-incubation with Chlamydia trachomatis lipopolysaccharide. Hum Reprod 20:2601-2607.

Evenson, D. and Wixon, R. (2006) Meta-analysis of sperm DNA fragmentation using the sperm chromatin structure assay. Reprod Biomed Online 12:466-472.

Gallon, F., Marchetti, C., Jouy, N. and Marchetti, P. (2006) The functionality of mitochondria differentiates human spermatozoa with high and low fertilizing capability. Fertil Steril 86:1526-1530.

Gorczyca, W., Traganos, F., Jesionowska, H. and Darzynkiewicz, Z. (1993) Presence of DNA strand breaks and increased sensitivity of DNA in situ denaturation in abnormal human sperm cells: analogy to apoptosis of somatic cells. Exp Cell Res 207:202-205.

Grunewald, S., Paasch, U., Said, T.M., Sharma, R.K., Glander, H.J. and Agarwal, A. (2005) Caspase activation in human spermatozoa in response to physiological and pathological stimuli. Fertil Steril 83:1106-1112. 
Henkel, R., Hajimohammad, M., Stalf, T., Hoogendijk, C., Mehnert, C., Menkveld, R., et al. (2004) Influence of deoxyribonucleic acid damage on fertilization and pregnancy. Fertil Steril 81:965-972.

Henkel, R., Kierspel, E., Hajimohammad, M., Stalf, T., Hoogendijk, C., Mehnert, C., et al. (2003a) DNA fragmentation of spermatozoa and assisted reproduction technology. Reprod Biomed Online 7:477-484. Henkel, R., Kierspel, E., Stalf, T., Mehnert, C., Menkveld, R., Tinneberg, H., et al. (2005) Effect of reactive oxygen species produced by spermatozoa and leukocytes on sperm functions in non-leukocytospermic patients. Fertil Steril 83:635-642.

Henkel, R., Ludwig, M., Schuppe, H.C., Diemer, T., Schill, W.B. and Weidner, W. (2006) Chronic pelvic pain syndrome/chronic prostatitis affect the acrosome reaction in human spermatozoa. World J Urol 24:39-44.

Henkel, R., Maass, G., Hajimohammad, M., Menkveld, R., Stalf, T., Villegas, J., et al. (2003b) Urogenital inflammation: Changes of leucocytes and ROS. Andrologia 35:309-313.

Henkel, R., Maass, G., Jung, A., Haidl, G., Schill, W.B. and Schuppe, H.C. (2007) Agerelated changes in seminal polymorphonuclear elastase in men with asymptomatic inflammation of the genital tract. Asian J Androl 9:299-304.

Henkel, R.R. (2011) Leukocytes and oxidative stress: dilemma for sperm function and male fertility. Asian J Androl 13:43-52.

Kasai, T., Ogawa, K., Mizuno, K., Nagai, S., Uchida, Y., Ohta, S., et al. (2002) Relationship between sperm mitochondrial membrane potential, sperm motility, and fertility potential. Asian J Androl 4:97-103.

Koppers, A.J., De Iuliis, G.N., Finnie, J.M., McLaughlin, E.A. and Aitken, R.J. (2008) Significance of mitochondrial reactive oxygen species in the generation of oxidative stress in spermatozoa. J Clin Endocrinol Metab 93:3199-3207.

Lopes, S., Sun, J.G. and Jurisicova, A. (1998) Sperm DNA fragmentation is increased in poor quality semen samples and correlates with failed fertilization in ICSI. Fertil Steril 69:528-532.

MacLeod, J. (1943) The role of oxygen in the metabolism and motility of human spermatozoa. Am J Physiol 138:512-518.

Marchetti, C., Obert, G., Deffosez, A., Formstecher, P. and Marchetti, P. (2002) Study of mitochondrial membrane potential, reactive oxygen species, DNA fragmentation and cell viability by flow cytometry in human sperm. Hum Reprod 17:1257-1265.

Martin, G., Sabido, O., Durand, P. and Levy, R. (2005) Phosphatidyl-serine externalization in human sperm induced by calcium ionophore A23187: relationship with apoptosis, membrane scrambling and the acrosome reaction. Hum Reprod 20:3459-3468.

Myhre, O., Andersen, J.M., Aarnes, H. and Fonnum, F. (2003) Evaluation of the probes 2'7'dichlorofluorescin, luminol, and lucigenin as indicators of reactive oxygen species formation. Biochem Pharmacol65:1575-1582.

Nicholson, D.W. (1999) Caspase structure, proteolytic substrates, and function during apoptotic cell death. Cell Death Differ 6:1028-1042.

Ochsendorf, F.R. (1999) Infections in the male genital tract and reactive oxygen species. Hum Reprod Update 5:399-420. 
Oehninger, S., Morshedi, M., Weng, S.L., Taylor, S., Duran, H. and Beebe, S. (2003) Presence and significance of somatic cell apoptosis markers in human ejaculated spermatozoa. Reprod Biomed Online 7:469-476.

Oosterhuis, G.J., Mulder, A.B., Kalsbeek-Batenburg, E., Lambalk, C.B., Schoemaker, J. and Vermes, I. (2000) Measuring apoptosis in human spermatozoa: a biological assay for semen quality? Fertil Steril 74:245-250.

Paasch, U., Grunewald, S., Agarwal, A. and Glander, H.J. (2004) Activation pattern of caspases in human spermatozoa. Fertil Steril 81:802-809.

Pasqualotto, F.F., Sharma, R.K., Potts, J.M., Nelson, D.R., Thomas, A. J. and Agarwal, A. (2000) Seminal oxidative stress in patients with chronic prostatitis. Urology 55:881885 .

Print, C.G. and Loveland, K.L. (2000) Germ cell suicide: new insights into apoptosis during spermatogenesis. Bioessays 22:423-430.

Quinn, P., Kerin, J.F. and Warnes, G.M. (1985) Improved pregnancy rate in human in vitro fertilization with the use of a medium based on the composition of human tubal fluid. Fertil Steril 44:493-498.

Raha, S. and Robinson, B.H. (2000) Mitochondria, oxygen free radicals and aging. Trends Biochem Sci 25:502-508.

Ricci, G., Perticarari, S., Fragonas, E., Giolo, E., Canova, S., Pozzobon, C., et al. (2002) Apoptosis in human sperm: its correlation with semen quality and the presence of leukocytes. Hum Reprod 17:2665-2672.

Sakkas, D., Mariethoz, E. and St John, J.C. (1999) Abnormal sperm parameters in humans are indicative of an abortive apoptotic mechanism linked to the Fas-mediated pathway. Exp Cell Res 251:350-355.

Sakkas, D., Moffatt, O., Manicardi, G.C., Mariethoz, E., Tarozzi, N. and Bizzaro, D. (2002) Nature of DNA damage in ejaculated human spermatozoa and the possible involvement of apoptosis. Biol Reprod 66:1061-1067.

Schaeffer, A.J. (2003) Epidemiology and demographics of prostatitis. Andrologia 35:252257.

Shekarriz, M., Thomas, A.J. and Agarwal, A. (1995) Incidence and level of seminal reactive oxygen species in normal men. Urology 45:103-107.

Sun, J.G., Jurisicova, A. and Casper, R.F. (1997) Detection of deoxyribonucleic acid fragmentation in human sperm: Correlation with fertilization in vitro. Biol Reprod 56:602-607.

Tremellen, K. (2008) Oxidative stress and male infertility - A clinical perspective. Hum Reprod Update 14:243-258.

Villegas, J., Schulz, M., Soto, L. and Sanchez, R. (2005) Bacteria induce expression of apoptosis in human spermatozoa. Apoptosis 10:105-110.

Wang, X., Sharma, R.K., Sikka, S.C., Thomas, A.J., Falcone, T. and Agarwal, A. (2003) Oxidative stress is associated with increased apoptosis leading to spermatozoa DNA damage in patients with male factor infertility. Fertil Steril 80:531-535.

Weidner, W., Krause, W. and Ludwig, M. (1999) Relevance of male accessory gland infection for subsequent fertility with special focus on prostatitis. Hum Reprod Update 5:421-432. 
World Health Organization (1999) WHO Laboratory Manual for the Examination of Human Semen and Sperm-cervical Mucus Interaction. Cambridge University Press, Cambridge, United Kingdom.

Yanushpolsky, E.H., Politch, J.A., Hill, J.A. and Anderson, D.J. (1996) Is leukocytospermia clinically relevant? Fertil Steril 66:822-825.

Zorn, B., Ihan, A., Kopitar, A.N., Kolbezen, M., Sesek-Briski, A. and Meden-Vrtovec, H. (2010) Changes in sperm apoptotic markers as related to seminal leukocytes and elastase. Reprod Biomed Online 21:84-92. 